Arthur Benz

\title{
Eine Gestalt, die alt geworden ist?
}

\author{
Thesen zum Wandel des Staates
}

\section{Einleitung}

Totgesagte leben länger. Dieses Sprichwort scheint auch auf den Staat zuzutreffen. Immer wieder wurde sein Absterben angekündigt, immer wieder wurde er als Herrschaftsform im Niedergang betrachtet. Der Staat wurde von Theoretikern zu unterschiedlichen Zeiten als eine alte, zu überwindende Gestalt bezeichnet, angefangen von Hegel über Marx, Engels und Lenin bis hin zu konservativen Staatswissenschaftlern des 20. Jahrhunderts. ${ }^{1}$ Historiker wie Wolfgang Reinhard haben seinen Aufstieg und Niedergang beschrieben, ${ }^{2}$ und auch in der Staatsrechtslehre der Bundesrepublik finden sich Abgesänge auf den Staat. ${ }^{3}$ Angesichts der globalen Vernetzung von Wirtschaft und Gesellschaft beobachteten Sozialwissenschaftler die Auflösung des Nationalstaats, erkannten einen neuen Feudalismus der internationalen Politik ${ }^{4}$ oder sahen eine postnationale Konstellation heraufdämmern. ${ }^{5}$ Inzwischen sprechen jedoch manche von einer Rückkehr des Staates, ${ }^{6}$ der demnach wieder an Bedeutung zu gewinnen scheint. Träfe dies zu, dann hätten sich Propheten, die noch vor nicht allzu langer Zeit sein »Absterben « verkündet hatten, wieder einmal geirrt.

Die Aufstiegs- und Niedergangsthesen leiteten sich in der Regel entweder aus Staatsbegriffen ab, die die Monopolisierung von Machtmitteln und Souveränität herausstellen, oder aus Gesellschaftstheorien, die den Staat als abhängig von gesellschaftlichen Entwicklungen betrachten und ihm Autonomie und Steuerungsfähigkeit absprechen. Diese Staatsbegriffe und -theorien sind jedoch wenig erhellend, weil sie von vornherein den Blick auf spezifische Phänomene verengen, die sich als historisch wandelbar erweisen oder die nur bestimmte Merkmale betreffen. Hierauf gestützte Aussagen über den Staat und seinen Wandel sind einseitig und verkürzen die Wirklichkeit auf partielle Aspekte. Allein wenn man den Wandel als normal betrachtet, ist es verfehlt, die Entwicklung des Staates als Zyklus von Aufstieg und Niedergang zu beschreiben. Ebenso verfehlt ist es daher auch, nunmehr von einer Rückkehr des Staates zu sprechen, weil man damit weiterhin dem zyklischen Modell

1 Zusammenfassend Benz 2008, S. 259-264.

2 Reinhard 1999.

3 Zusammenfassend Möllers 2008, S. 44-47.

4 Bull 1977.

5 Habermas 2006; Leibfried, Zürn 2006 a.

6 Beispielsweise Heinze 2009; James 2009.

Leviathan, 40. Jg., 2/2012, S. $223-2477$ 
folgt. In all diesen Analysen wird die Stabilität, aber auch die Anpassungsfähigkeit des Staates unterschätzt, wenn nicht gar ignoriert, kommt die Mehrdimensionalität des Wandels jedenfalls nicht in den Blick.

Mit der Diskussion über den vermeintlichen Niedergang des Staates verbunden war auch ein Niedergang der Staatstheorie, ${ }^{7}$ der durch das neuerliche Interesse am Staat nicht revidiert wurde. Aussagen beruhen weitgehend auf Beobachtungen und Beschreibungen, ohne dass der Staatsbegriff geklärt wird oder die Ursachen von Wandel angemessen analysiert werden. In der Regel findet man Staatsbegriffe, die, wie Gunnar Folke Schuppert zu Recht kritisiert, einen bestimmten Typus von Staat betreffen, aber dabei die Verschiedenheit der Staatstypen und seine Wandlungsfähigkeit unberücksichtigt lassen. ${ }^{8}$ Es wundert einen daher nicht, dass Abhandlungen bei der Beschreibung des Staates manchmal ins Metaphorische abgleiten. Die Staatstheorie ist also weniger » auf der Suche nach ihrem Gegenstand « 9 , vielmehr erfordert der Gegenstand die Suche nach einer Theorie.

Nicht zuletzt mit Blick auf die aktuelle Diskussion über den Staat, die durch die jüngste Wirtschaftskrise angestoßen wurde, scheint es erforderlich zu sein, die Frage zu klären, was es mit dem Wandel des Staates auf sich hat und wodurch er verursacht ist. Mit Wandel bezeichne ich dabei Veränderungen, die durch bestimmte Mechanismen bewirkt werden und dauerhafte Folgen nach sich ziehen. Sie sind also von zufälligen oder situativen Veränderungen (etwa der Privatisierungspolitik einer neoliberalen Regierung) zu unterscheiden. Um den Wandel der Gestalt des Staates besser zu verstehen, muss zunächst geklärt werden, was unter Staat zu verstehen ist, um dann die Bestimmungsfaktoren des Wandels und dessen Folgen zu analysieren. Es stellt sich also die Frage, was sich wandelt, warum dies der Fall ist und wie sich der Wandel vollzieht. Dementsprechend will ich zunächst den Staatsbegriff präzisieren, dann - in knapper Auseinandersetzung mit Staatstheorien - die Wirkungsmechanismen des Wandels ermitteln, um, hierauf gestützt, Thesen zum aktuellen Wandel des Staates skizzieren zu können.

Dabei ist vorab zu betonen, dass sich die im Folgenden präsentierten Überlegungen auf den » modernen « Staat beziehen, also auf jene Form von Herrschaft, die sich im Europa der Neuzeit gegen alternative Herrschaftsformen wie die Feudalordnung, große Reiche oder Städtebündnisse durchsetzte, ${ }^{10}$ die politische Entwicklung des "Westens " prägte ${ }^{11}$ und sich in einer langen, an Varianten reichen Geschichte schließlich zu der Form entwickelte, die heute üblicherweise als demokratischer und

7 Roth 2003.

8 Schuppert 2009, S. 9-11.

9 Offe 1987.

10 Spruyt 1994.

11 Winkler 2009. 
sozialer Rechtsstaat bezeichnet wird. ${ }^{12}$ Ferner geht es um eine empirische Theorie des Staates, nicht um eine normative. Ich behaupte weder, dass der Staat oder eine bestimmte Form des Staates gut oder geeignet ist, gesellschaftliche Probleme zu lösen, noch stelle ich die Frage, ob der Staat tatsächlich legitime Herrschaft gewährleistet. Eine fundierte Analyse des Wandels von Staatlichkeit deckt allerdings inkonsistente Entwicklungen und Spannungen auf, die die Funktionsweise des demokratischen und sozialen Rechtsstaates gefährden können und daher besonderer Aufmerksamkeit einer Staatskritik bedürfen.

\section{Was wandelt sich? - Zum Staatsbegriff}

Der Staatsbegriff gehört zu jenen Begriffen in den Sozialwissenschaften, die ein mehrdimensionales Phänomen erfassen und deswegen nicht eindeutig, sondern immer nur aus einer bestimmten Perspektive definiert werden können. »Was der Staat ist, läßt sich nicht auf einen einzigen Begriff bringen oder in einer schulmäßigen Definition einfangen. «13

Diese Mehrdimensionalität des Begriffs wird in der Diskussion über den Wandel des Staates bzw. der Staatlichkeit nicht immer angemessen berücksichtigt. Viele Beiträge beziehen sich primär auf die »Staatsgewalt", sie konzentrieren sich damit auf die Handlungsfähigkeit von Regierungen oder Verwaltungen. Das gilt nicht zuletzt für neuere Analysen zur vermeintlichen Rückkehr des Staates in der Finanzkrise. ${ }^{14}$ Andere Thesen betreffen den Wandel von Staatsfunktionen, wobei meistens nicht deutlich wird, wie der Funktionswandel vom normalen Politikwandel abzugrenzen ist. So wird oft ein Niedergang des Sozialstaates konstatiert, ohne zu unterscheiden, ob Regierungen mit Reformen in der Sozial- und Arbeitsmarktpolitik auf dauerhafte demographische Entwicklungen und Veränderungen in der Arbeitswelt reagieren oder ob Leistungskürzungen mit der Programmatik einer Regierungspartei zu erklären sind. Andere Studien konstruieren einen bestimmten Entwicklungsstand des Staates als Bezugspunkt des Wandels, je nach Erkenntnisinteresse variierend: So blicken Historiker und Juristen auf den souveränen Nationalstaat des 19. Jahrhunderts oder den Machtstaat des frühen 20. Jahrhunderts, ${ }^{15}$ während sozialwissenschaftliche Analysen sich auf den Wohlfahrtsstaat oder den demokra-

12 Damit ist keine Einschränkung auf ein "goldenes Zeitalter « des Staates verbunden, vielmehr soll der Staatsbegriff gegenüber ubiquitären Verwendungen präzisiert werden. Weder schließt er etwa den mittelalterlichen »Ständestaat « noch den antiken »Stadtstaat « ein, und auch autoritäre Regime sollen nicht berücksichtigt werden. Selbstverständlich sollte auch nicht übersehen werden, dass der Typus des modernen Staates in unterschiedlichen Ausprägungen entstanden ist und existiert. Ich gehe aber davon aus, dass die im Folgenden definierten Merkmale westliche Staaten - wenn auch mehr oder weniger - zutreffend beschreiben.

13 Isensee 1995, S. 134.

14 Mayntz 2010.

15 Vgl. de Jouvenel 1989; Reinhard 1999. 
tischen Rechts- und Interventionsstaat beziehen, dessen "goldenes Zeitalter " sie auf die ersten Jahrzehnte nach dem Zweiten Weltkrieg datieren. ${ }^{16}$

Diese selektiven Betrachtungen blenden eine Dimension des Staatsbegriffs aus, die nicht nur dessen Kerngehalt ausmacht, sondern für das Verständnis des Wandels essentiell ist. Denn Wandel des Staates darf weder mit dem Niedergang einer spezifischen Ausprägung der Staatlichkeit gleichgesetzt werden, die er im Laufe seiner Geschichte angenommen hat, noch darf er mit Politikwandel verwechselt werden, der in einer Demokratie normal ist. Er meint dauerhafte Veränderungen der Herrschaftsordnung, die wir als Staat bezeichnen. Um diese Unterscheidung in den Blick zu bekommen, muss man die Tatsache in den Mittelpunkt rücken, dass der moderne Staat eine institutionelle Ordnung bildet. Er entstand bekanntlich in einem langen historischen Prozess, der mit dem Zerfall der mittelalterlichen Ordnung einsetzte. Man hat seine Entstehung häufig als Prozess der Machtakkumulation in einem Zentrum beschrieben, der in den einzelnen Gebieten Europas infolge geopolitischer Gegebenheiten unterschiedlich weit ging und mehr oder weniger große Widerstände der Stände überwinden musste. ${ }^{17}$ Entscheidend für die Herausbildung des modernen Staates war aber die Institutionalisierung von Herrschaft. Sie beseitigte Willkür durch Personen und ermöglichte wirksames Regieren bei gleichzeitiger Begrenzung von Macht durch Rechtsnormen und durch die Machtaufteilung auf konkurrierende Organe. ${ }^{18}$

Die Dualität von Schaffung und Begrenzung von Macht, die mit der Institutionalisierung von Herrschaft erreicht wurde, wird vernachlässigt, wenn man das Wesen des Staates in der Souveränität sieht. Der Souveränitätsbegriff entspricht einer spezifischen Staatstheorie aus einer bestimmten historischen Phase, die in der Ideengeschichte auch keineswegs unumstritten war. ${ }^{19}$ Im Konzert der Mächte konnten Staaten zwar Souveränität beanspruchen, aber sie erreichten stets nur eine relative Souveränität und Handlungsautonomie, die von anderen Staaten oder gesellschaftlichen Kräften im Staat anerkannt werden musste und dadurch eingeschränkt wurde. ${ }^{20}$ Wenn heute ein »Souveränitätsverlust « des Staates konstatiert wird, dann ist meistens nicht deutlich, dass dieser stets an einem theoretischen Konstrukt gemessen wird. Der Blick auf die historische Realität relativiert einen solchen vermeintlichen Verlust erheblich. Ähnliches gilt für das Konzept des Gewaltmonopols, das schon Max Weber mit dem Adjektiv » legitim « versah, um auszudrücken, dass das exklusive Recht des Staates zur physischen Gewaltsamkeit nur Geltung erlangt, wenn die Beherrschten es anerkennen und wenn es durch Regeln beschränkt ist. ${ }^{21}$ Wenn in der Geschichtsschreibung also der alle Lebensbereiche durchdringende »Machtstaat", wie ihn Europa in der ersten Hälfte des 20. Jahrhunderts erlebte, als Höhe-

16 Leibfried, Zürn 2006 b.

17 Zum Beispiel Elias 1976; Tilly 1990.

18 Vgl. dazu Benz 2008, S. 17-38.

19 Grimm 2009; Kriele 2003.

20 Keohane 2000.

21 Weber 1976, S. 29. 
punkt der Staatsentwicklung dargestellt wird, ${ }^{22}$ von dem aus der Abstieg begann, dann wird verkannt, dass dieser Staat eigentlich vom Typus des » modernen « Staates abwich und er per se instabil war. Denn im modernen Staat wird die Ausübung physischer Gewaltsamkeit zwar monopolisiert, jedoch unter der Bedingung, dass sie gleichzeitig durch Berechenbarkeit und Zustimmung der Beherrschten begrenzt ist.

Als Institutionenordnung stellt der Staat ein Regelsystem dar, dessen wesentliche Merkmale durch Außengrenzen und Binnendifferenzierung zu beschreiben sind. Mit der institutionellen Verankerung einer »äußeren Form « und einer »inneren Form « konstituiert und beschränkt der moderne Staat Herrschaft. Mit dieser Unterscheidung lässt sich der Staat nach seinen wesentlichen Merkmalen definieren, man gewinnt zugleich, wie im Folgenden zu zeigen ist, einen analytischen Rahmen, mit dem die den Wandel verursachenden und hemmenden Mechanismen genauer bestimmt werden können.

Nach außen erfolgt die Schaffung und Begrenzung staatlicher Herrschaft mit der Abgrenzung seines Machtbereichs, und zwar durch

- Gebietsgrenzen, die den Hoheitsbereich definieren, aber auch gegenüber anderen Staaten abgrenzen;

- die Festlegung, wer zu den Staatsbürgern gehört, also als Mitglied einer Nation an der Herrschaft partizipieren kann, und durch den gleichzeitigen Ausschluss von »Ausländern « von Partizipationsrechten;

- die Zuschreibung von spezifischen Funktionen, die im Staat erfüllt werden sollen, weil entsprechende Leistungen von anderen Modi kollektiven Handelns (Markt, Gemeinschaften) nicht erwartet werden können. ${ }^{23}$

Nach innen erfolgt die Schaffung und Begrenzung von Herrschaft durch institutionelle Differenzierung, und zwar primär

- durch Schaffung einer Verfassung, die Regeln der Herrschaftsordnung enthält, die bei der Herrschaftsausübung nicht zur Disposition gestellt werden dürfen (Differenzierung von Verfassung und verfasster Politik);

- durch Bindung aller Gesetzgebung an die Zustimmung des Volkes bzw. seiner Vertreter (Demokratie, das heißt Differenzierung zwischen Regierenden und dem »Demos «);

- durch Übertragung der ausführenden Gewalt auf eine Bürokratie, die an Gesetze gebunden ist und durch unabhängige Gerichte kontrolliert wird, also durch Differenzierung zwischen Gesetzgebung, Exekutive und Judikative.

Wenn so der Staat als institutionelle Ordnung verstanden werden kann, die kollektive Macht schafft und begrenzt, dann macht es offensichtlich wenig Sinn, seinen Wandel als Aufstieg und Niedergang zu beschreiben. Zum einen können diese Pro-

22 Siehe etwa de Jouvenel 1989; Reinhard 1999.

23 Siehe dazu Benz 2008, S. 122-135. In Abweichung von der auf Georg Jellinek (1905, S. 381-420) zurückzuführenden Trias von Merkmalen des Staates verwende ich nicht den Begriff Staatsgewalt. Gegenüber anderen Modi gesellschaftlicher Handlungskoordinierung ist der Staat durch seine Funktionen abzugrenzen. Die "Staatsgewalt « ist durch Differenzierung seiner inneren Form beschränkt, was in einem Begriff des modernen Staates (auf den sich auch der Staatsbegriff Jellineks bezieht) erfasst werden muss. 
zesse des Wandels ja dem Austarieren von Machtbalancen zwischen Staaten oder Staat und gesellschaftlichen Akteuren dienen. Zum anderen ist davon auszugehen, dass sich Wandel in den einzelnen Bereichen der institutionellen Ordnung unterschiedlich vollzieht, wir insgesamt also einen komplexen, mehrschichtigen Prozess der Veränderung beschreiben müssen. Tatsächlich zeigt die im Folgenden dargestellte Analyse, dass der Wandel des Staates die einzelnen Bereiche der Institutionenordnung unterschiedlich erfasst und ungleichzeitig verläuft, was Spannungen und Konflikte zur Folge hat, die wiederum eine fortdauernde Dynamik erzeugen.

\section{Erklärung des Wandels - Zur Staatstheorie}

In der mangelnden Komplexität der analytischen Konzepte liegt das zentrale Problem der Staatstheorien, die sich mit Ursachen des Wandels befassen.

Soziologische Staatstheorien konzentrieren sich auf das Verhältnis von Staat und Gesellschaft. Als treibende Kräfte der Entwicklung und des Wandels des Staates gelten die Dynamik der ökonomischen Verhältnisse, Prozesse der gesellschaftlichen Differenzierung oder Veränderungen von kulturellen (religiösen) Normen bzw. Ideen. Erstere werden von neomarxistischen Theorien betont. Sie unterstellen, dass der Staat von ökonomischen Ressourcen und Massenloyalität abhängig sei, was ihn, um Klassenkämpfe abzuwenden, zu einer Ausweitung von Wohlfahrtsleistungen zwinge, ihn aber zugleich anfällig für krisenhafte Entwicklungen in der kapitalistischen Gesellschaft mache. ${ }^{24}$ Systemtheoretiker betrachten den Staat dagegen als autonomes Teilsystem der Gesellschaft, das wegen der »Selbstreferenzialität von Systemen « nur begrenzt steuern könne, jedoch spezifische gesellschaftliche Funktionen erfülle. Wandel des Staates resultiere aus Prozessen der gesellschaftlichen Differenzierung, die zu einer Ausweitung und Umdefinition von Staatsfunktionen ${ }^{25}$ bzw. einer Veränderung von Selbstbeschreibungen des politischen Systems führten. ${ }^{26}$ Die Wirkungen von Weltanschauungen (Religion) und Denktraditionen (Ideen) stellen vor allem international vergleichende Arbeiten heraus, ${ }^{27}$ während Michael Mann die Staatsentwicklung als Ergebnis eines kontingenten Zusammenwirkens von militärischer, ökonomischer, ideologischer und politischer Macht erklärt. ${ }^{28}$

Gesellschaftszentrierte Erklärungen des Wandels werfen zwei fundamentale Probleme auf. Zum einen lassen sich auf der Makroebene der Gesellschaft Mechanismen, die Wandel verursachen, nur sehr grob benennen. Deshalb eignen sich diese Theorien nicht, wenn man ermitteln will, wie und in welcher Weise diese Mechanismen die einzelnen Bereiche der institutionellen Ordnung des Staates beeinflussen und verändern. Es ist angesichts unterschiedlicher Theorien auch zunehmend unklar, wie die gegenwärtige Gesellschaft zu beschreiben ist und welche Aspekte ihrer

\section{Zum Beispiel Offe 1972.}

25 Willke 1997.

26 Luhmann 1981.

27 Exemplarisch Dyson 1980.

28 Mann 1993. 
Entwicklung den Staat betreffen. Es wird sogar bezweifelt, ob die Soziologie »eine Gesellschaft entdecken kann, der die Ordnung des Politischen entsprechen müsste «, ${ }^{29}$ da Staat und Gesellschaft sich weitgehend unabhängig voneinander entwickeln würden.

Institutionalistische Ansätze der Staatstheorie blenden den Wandel des Staates in der Regel aus. Zwar betrachten sie die Form des Staates als historisch bedingt, sie liefern aber keine überzeugende Erklärung für deren Veränderung. Dies gilt grundsätzlich auch für Vertreter des Institutionalismus in der amerikanischen Politikwissenschaft, die in den 1980er Jahren den Staatsbegriff für die vergleichende Politikforschung wiederentdeckten. ${ }^{30}$ Mit dem historischen Institutionalismus wollen Sozialwissenschaftler den Wandel erklären, allerdings sind in diesem Kontext bisher keine Beiträge zur Staatstheorie zu verzeichnen, sondern nur Studien zu einzelnen Staaten. ${ }^{31}$ Historisch orientierte Gesellschaftstheoretiker wie etwa Claus Offe machten die institutionalistische Wende mit und entdeckten die »relative Autonomie" des Staates in einer zunehmend desorganisierten Gesellschaft. Der Staat gilt daher nicht mehr als "gesellschaftlich bedingt " oder durch sozio-ökonomische Verhältnisse determiniert, sondern als treibende Kraft der gesellschaftlichen Entwicklung. Konsequenterweise erklärten sie, dass der Wandel von Staatlichkeit durch den Staat selbst verursacht sei. ${ }^{32}$ In diesem Sinne sprechen auch Vertreter des Bremer SFB "Staatlichkeit im Wandel « von einer »Selbsttransformation des Staates «. ${ }^{33}$ Dessen Wandel wird nunmehr in den Institutionen verortet, und politische Prozesse im Staat gelten als ursächlich für den Wandel. Wie sich Institutionen wandeln, welche Akteure und Interessen dabei relevant sind, welche Restriktionen auftreten und ob bzw. wie diese überwunden werden, wird dabei aber nicht hinreichend geklärt.

Theorien internationaler Integration (Funktionalismus, Intergouvernementalismus, Neorealismus ${ }^{34}$ scheinen insofern weiterzuführen, als sie die Transformation des Staates durch die Internationalisierung von Staatstätigkeit erklären, ohne dabei eine Auflösung des Staates zu unterstellen. Allerdings blenden sie den damit verbundenen internen Wandel aus. Untersuchungen zur Europäisierung wiederum konzentrieren sich bisher auf Politikfelder oder einzelne Institutionen wie Regierungen oder Parlamente, sie erfassen den institutionellen Wandel des Staates nur partiell.

Generell ist in Staatstheorien eine Kluft zwischen gesellschaftszentrierten und institutionalistischen Ansätzen festzustellen. Sie kann überwunden werden, wenn man von der analytischen Unterscheidung von äußerer und innerer Form ausgeht. Einen wichtigen Beitrag hierzu lieferten Stefano Bartolini und Maurizio Ferrera in ihren

29 Wagner 1996.

30 Evans et al. 1985.

31 Beispielsweise Orren, Skowronek 2004

32 Offe 1996.

33 Hurrelmann et al. 2008, S. 38.

34 Vgl. Wiener, Diez 2009. 
historischen Analysen der europäischen Integration. ${ }^{35}$ Beide griffen die Theorie der politischen Strukturierung auf, mit der Stein Rokkan die Entstehung unterschiedlicher Ausprägungen von Staatlichkeit erklärte. Dieser Ansatz erweist sich als höchst fruchtbar, um den Wandel des Staates zu analysieren. Zum einen beruht er auf einer Theorie kollektiven Handelns, erfasst somit soziale Mechanismen, durch die gesellschaftliche Entwicklungen in die politischen Strukturen hineinwirken. Damit kann der Zusammenhang zwischen Gesellschaft und Staat erfasst werden, ohne angeben zu müssen, in welcher Gesellschaft wir uns befinden. Zum anderen lassen sich die Mechanismen auf spezifische Strukturmerkmale des Staates beziehen. Allerdings erklären diese Theorien nur Auswirkungen auf die äußere Form der staatlichen Institutionenordnung. Wir müssen sie daher ergänzen durch eine Theorie, welche den Wandel interner Strukturen erklärt. Hierbei helfen uns Institutionentheorien. Akteurszentrierten Institutionentheorien zufolge resultiert Wandel aus Interaktionen von Amtsinhabern, die Macht- und Ressourcenverteilungen ändern, um ihre besonderen Interessen besser durchsetzen zu können, und die Regeln kollektiven Handelns modifizieren, wenn sie nicht mehr geeignet sind, um Verteilungen zu legitimieren oder erforderliche Entscheidungen zu treffen. Solche Veränderungen werden aber erst dauerhaft wirksam, wenn sie in Reformen formaler Regeln oder in expliziten oder impliziten Vereinbarungen verbindlich festgelegt werden. Wie neuere Theorien des historischen Institutionalismus ${ }^{36}$ zeigen, beschränken Transformationskosten die Reichweite institutioneller Reformen und des Wandels. Diese Kosten steigen mit zunehmender Komplexität bestehender Binnenstrukturen und der in ihnen verfestigten Machtverteilungen. Deshalb erfasst kein Wandel jemals in umfassender und kohärenter Weise die gesamte Institutionenordnung des Staates, sondern immer nur einzelne Bereiche und zu verschiedenen Zeitpunkten. Prozesse des Wandels verlaufen in unterschiedlichen Sequenzen und können zu entsprechenden Strukturbrüchen führen. Das gilt nicht nur für revolutionäre Transformationen von Staaten, sondern genauso für den fortlaufenden Wandel des modernen Staates westlicher Prägung, der hier betrachtet wird. Im Folgenden will ich diese theoretische Perspektive näher erläutern. Aus ihr lassen sich Erkenntnisse darüber gewinnen, wie sich der Staat aktuell wandelt. Im vorliegenden Zusammenhang können reale Entwicklungen selbstverständlich nur grob skizziert werden.

\section{Aspekte des Wandels - Dynamiken, ungleichzeitige Entwicklungen und Strukturbrüche}

Wenn hier, wie oben vorgeschlagen, die staatliche Institutionenordnung nach Merkmalen einer äußeren und einer inneren Form bestimmt wird, sind damit einerseits zentrale Voraussetzungen für das Ausbalancieren von Machtschaffung und Machtbegrenzung genannt. Andererseits ist damit aber auch eine Unterscheidung getroffen, die gesellschaftszentrierte und institutionalistische Erklärungen des Prozesses

35 Bartolini 2005; Ferrera 2005.

36 Falletti, Lynch 2009; Immergut 1998; Liebermann 2002; Mahoney 2000; Pierson 2004. 
des Wandels miteinander verbinden kann. Wie im Folgenden näher erläutert wird, induzieren gesellschaftliche Entwicklungen unmittelbar Veränderungen der äußeren Form, das heißt der territorialen Abgrenzung von Herrschaft, der Definition des Staatsvolks und der Bestimmung von Staatsfunktionen, während die innere Form, also Verfassung, Demokratie und Bürokratie, primär durch politische Prozesse des Wettbewerbs um Macht und der Verhandlung um Reformen verändert wird. Dies bedeutet nicht, dass erstere gesellschaftlich determiniert wären. Selbstverständlich resultiert die staatliche Institutionenordnung in allen Dimensionen aus politischen Entscheidungen. Dabei können Akteure, die in Staaten Herrschaft ausüben, die Verfassung, Demokratie und Bürokratie selbst ändern, weil sie über die erforderlichen Kompetenzen verfügen, während sie das Territorium, die Nation und Staatsfunktionen nicht allein bestimmen können. Daher treffen die gesellschaftlichen Dynamiken, die sich in der äußeren Form des Staates bemerkbar machen, auf relativ starre institutionelle Strukturen (Gebietsstrukturen, Nationalitäten, Kompetenzen der Staatsgewalt), während die innere Form durch eine schwächere politische Dynamik, aber flexible Strukturen gekennzeichnet ist.

\section{Dynamik der Gesellschaft und Veränderungen der äußeren Form des Staates}

Obgleich westliche Gesellschaften durch zunehmende ökonomische Disparitäten und funktionale Differenzierung geprägt sind, lassen sich gesellschaftliche Dynamiken heute nicht mehr durch Klassenstrukturen oder die Eigenlogik von Systemen erklären, vielmehr sind sie auf das Zusammenwirken korporativer oder individueller Akteure zurückzuführen. Mechanismen, welche die Wechselwirkung von Gesellschaft und Staat erzeugen, können daher besser mit akteurszentrierten Ansätzen erfasst werden, wie sie in den erwähnten Arbeiten von Stein Rokkan ${ }^{37}$ und Stefano Bartolini ${ }^{38}$ zu finden sind. Beide Autoren unterschieden in Anlehnung an Albert O. Hirschman drei Typen von kollektiven Handlungen, mit denen Akteure auf Herrschaft reagieren können, nämlich Austritt/Eintritt (exit/entry), Loyalität (loyalty) und Widerspruch (voice). Sie interpretierten diese drei Handlungsmuster als soziale Mechanismen, die Strukturen verändern, wobei sich Austritt/Eintritt auf territoriale Strukturen, Loyalität auf Formen der Gemeinschaftsbildung und Widerspruch auf Strukturen der Interessenorganisation beziehen. Wie schon Bartolini ${ }^{39}$ andeutete, betreffen sie die äußere Form des Staates. Mittels dieser analytischen Kategorien lassen sich Erkenntnisse über deren Veränderung infolge gesellschaftlicher Prozesse gewinnen. Die grundlegenden Tendenzen können wie folgt skizziert werden:

(1) Staatsgrenzen beruhen auf einer doppelten Anerkennung, nämlich durch die Bürgerschaft im jeweiligen Staat und durch Regierungen anderer Staaten. Im Verlauf des letzten Jahrhunderts haben Staatsgebiete relativ stabile Formen angenommen, weil gewaltsame Änderungen gegen das Völkerrecht verstoßen, dessen Anerkennung ein Merkmal des modernen Staates geworden ist. Fusionen und Sezessio-

37 Rokkan 1999.

38 Bartolini 2005.

39 Ebd., S. 45.

Leviathan, 40. Jg., 2/2012 
nen sind Ausnahmen in einer Welt von Staaten, deren Territorium fixiert ist. Was sich geändert hat, sind die Wirkungen von Grenzen. ${ }^{40}$ Seit dem 19. Jahrhundert öffneten moderne Staaten die Grenzen ihrer Gebiete gezielt und selektiv für Transaktionen von Gütern und Kapital, ließen also kontrolliert Eintritts- und AustrittsOptionen zu. Je mehr diese Optionen wahrgenommen wurden, desto stärker gerieten Staaten in Konkurrenz um leistungsfähige Ressourcen und Unternehmen - mit der Folge, dass die Staatsgrenzen überschreitenden Transaktionen immer weiter zunahmen. Herrschaftsräume von Staaten sind daher weniger denn je territorial geschlossen, sondern offen und interdependent.

Heute bestehen Interdependenzen nicht nur wie in früheren Jahrhunderten in Warenaustausch- und Kommunikationsbeziehungen, die vorrangig der Chancenund Wohlstandsvermehrung dienten, sondern verstärkt auch in negativen externen Effekten, die etwa den sozialen Ausgleich im Wohlfahrtsstaat durch Lohnkürzungen und Absenkungen öffentlicher Leistungen gefährden und mit dem Umwelt- und Klimawandel oder dem internationalen Terrorismus bedrohliche Folgen zeigen. ${ }^{41}$ Auch hat die Mobilität von Personen, Gütern, Kapital und Information infolge des technischen Fortschritts, des Bevölkerungswachstums, eines höheren Wohlfahrtsund Bildungsniveaus, aber auch infolge der Zunahme von Krisen in vielen Teilen der Welt zugenommen und sich beschleunigt. Dadurch sind Staatsgrenzen als Element institutionalisierter Herrschaft zu einem Problem geworden. Personen und Gegenstände, auf die sich staatliche Regeln und Entscheidungen erstrecken, können sich diesen durch Grenzüberschreitungen leicht entziehen, und ebenso können Staaten viele ihrer Leistungen nicht mehr ohne weiteres auf ihre eigene Bevölkerung begrenzen, weil öffentliche Güter auch Ausländern zugänglich werden und Staaten für Zuwanderer sorgen müssen.

Die wachsende Mobilität hat bekanntlich Auswirkungen auf verschiedene Politikfelder, wie die Wirtschafts-, Steuer- Sozial- oder Umweltpolitik. Sie hat darüber hinaus auch Folgen für die Struktur des Staates, weil sie die Handlungsräume staatlicher Akteure sowie die Wirkungsräume staatlicher Tätigkeit verschiebt, also die Gewichte zwischen Ebenen des Regierens verlagert (»rescaling «). ${ }^{42}$ Wir beobachten zum einen eine Internationalisierung von Politik, die etwa in der Wirtschafts- und Umweltpolitik den Austritts-Strategien von Unternehmern und Kapitalbesitzern folgt. Zum anderen stellen wir aber auch eine Regionalisierung innerhalb staatlicher Territorien fest, die ebenfalls mit der Mobilität zusammenhängt, da zuwandernde Akteure (Einwanderer und Unternehmen) durch ihre Ansiedlungsstrategien neue räumliche Strukturen erzeugen. Doch weil sich institutionalisierte Grenzen des Staates, die dessen Kompetenz- und Legitimationsräume definieren, nicht ändern, erweist sich das Prinzip der »Gebietshoheit « immer mehr als Fiktion. Entscheidungen territorialer Einheiten werden weniger autonom getroffen und sind durch stärkere Interdependenzen geprägt, was Staaten zur Kooperation oder Kompetenzverlage-

40 Schimank 2005.

41 Zürn 1998, S. 75.

42 Vgl. Brenner 2004. 
rung auf größere Einheiten zwingt. Staaten können immer mehr ihrer Aufgaben nur noch partiell erfüllen und müssen dabei Effekte einkalkulieren, die in anderen Staaten verursacht werden. Aus der Welt der Territorialstaaten ist eine zunehmend verflochtene Staatenwelt geworden, deren Strukturen sich in Richtung auf ein Mehrebenensystem verändern.

(2) Eng verbunden mit den grenzüberschreitenden Transaktionen sind Veränderungen von Loyalitätsbeziehungen. Bartolini hat diese Prozesse als »system building « bezeichnet, womit er die Formierung von nationalen Identitäten meint. Im Zeitalter der Nationalstaaten diente, so seine These, die Schließung von territorialen Grenzen dazu, den nationalen Zusammenhalt zu stärken. Loyalität zwischen den Mitgliedern einer Nation war zudem Voraussetzung dafür, dass Umverteilung im Wohlfahrtsstaat von der Mehrheit der Bürger anerkannt wurde. ${ }^{43}$ Dieser sich selbst verstärkende Zusammenhang von Nation, Wohlfahrtsstaat und Demokratie wird in der mobilen Weltgesellschaft durch zwei Entwicklungen zunehmend gestört. Zum einen nimmt der Anteil der Menschen ohne Mitgliedschaftsrechte in einem Staat zu, weil eine neue Staatsbürgerschaft in der Regel erst nach mehr oder weniger langen Aufenthaltszeiten erworben werden kann. Ausländer sind den Gesetzen eines Staates unterworfen, tragen zum Wohlstand bei und profitieren von öffentlichen Gütern, verfügen aber nicht über dieselben politischen Rechte. Zum anderen wird, unabhängig von der formalen Staatsbürgerschaft, die Intensität der auf die Nation bezogenen Loyalität schwächer, sosehr auch Parteien die Förderung nationaler Integration postulieren. Manche Sozialwissenschaftler haben vermutet, dass Nationen, da sie Gemeinschaften bilden, sich in Individualisierungsprozessen auflösen würden. Aber das Bedürfnis von Menschen, in sozialen Kontexten Orientierungen für ihr Handeln, Anerkennung und Unterstützung zu finden, hat keineswegs abgenommen. Tatsächlich scheinen Loyalitäten gegenüber der abstrakten Staatsnation abzunehmen, jedoch Loyalitäten zu anderen Gemeinschaften an Gewicht zu gewinnen. Eine Folge dieser Entwicklung sind regionale Nationalismen, die in vielen Staaten seit Mitte der 1970er Jahre aufgekommen sind. Darüber hinaus entwickeln Menschen zunehmend Mehrfachidentitäten, die sich neben der Staatsnation auch auf regionale und lokale Einheiten wie auf transnationale Ebenen wie die EU beziehen.

Diese Verlagerungen von Identifikationen und die damit verbundenen Spannungen haben offensichtlich zur Folge, dass Nation - anders als in der Phase der Demokratisierung und der Entwicklung des Sozialstaats - wieder stärker durch kulturelle Merkmale definiert wird, während das Kriterium der politischen Beteiligung in den Hintergrund rückt. Die Forderung nach Gleichheit wird durch Betonung von Differenz überlagert. Unter diesen Bedingungen können Staaten eine homogene Nation weder durch Inklusion noch durch Exklusion herstellen, sondern müssen Pluralität organisieren und den Widerspruch zwischen Gleichheit und Differenz bewältigen. ${ }^{44}$ Die Staatsbürgernation erweist sich nicht mehr als homogene »Vergemeinschaftung " von Bürgern, sie wird zunehmend durchdrungen von » regionalen

43 Miller 1995.

44 Vgl. dazu Maíz, Requejo 2005.

Leviathan, 40. Jg., 2/2012 
Nationalismen « und von sozio-kulturellen Differenzierungen der sich entwickelnden Weltgesellschaft.

(3) Der dritte Mechanismus des Widerspruchs (voice) erklärt nach Stein Rokkan die Muster gesellschaftlicher Interessenorganisation in Parteien und Verbänden. Bartolini betont diese "politische Strukturierung " als die zentrale Voraussetzung von Demokratie. Richtig ist daran, dass Parteien und Verbände dazu beitragen, individuelle Willensäußerungen in kollektive Entscheidungen zu transformieren. Die jeweilige politische Strukturierung von Gesellschaften, die sich vor allem im Parteiensystem niederschlägt, erklärt, welche Erwartungen sich an die Leistungen des Staates richten werden, und lässt damit die funktionalen Abgrenzungen von Staat und Gesellschaft erkennen, die in jedem Land anders verlaufen.

Im modernen Staat wurde im Zeitalter der Industrialisierung - bei allen Variationen in einzelnen Ländern - die Konfliktlinie zwischen Kapital und Arbeit prägend für die Entwicklung des Parteiensystems, die in mitteleuropäischen Staaten durch eine starke christlich-konservative, soziale Leistungen befürwortende Partei überlagert wurde. Seit den 1980er Jahren drängten neo-konservative und liberale Parteien auf einen Rückbau des Wohlfahrtsstaates und eine Privatisierung öffentlicher Aufgaben. Die daraus entstandene Konfliktstruktur ist nicht verschwunden, inzwischen steht aber nicht mehr die Debatte um Verstaatlichung oder Privatisierung im Vordergrund, sondern um Modalitäten der Funktionsteilung zwischen Staat, Markt und sozialen Gemeinschaften. Ferner finden inzwischen postmaterialistische Werte und ökologische Themen stärkere Resonanz und bilden sich in Parteiensystemen ab. Darüber hinaus schließt die Frage nach den Staatsfunktionen mittlerweile die Frage nach der Ebene von Staatlichkeit ein. Marktschaffende und -regulierende Politik muss international koordiniert werden und findet in Europa seit der Errichtung des einheitlichen Marktes hauptsächlich im Rahmen der EU statt. Öffentliche Leistungen werden dagegen national oder auf subnationaler Ebene erbracht, allerdings unter Bedingungen eines verstärkten Wettbewerbs, wodurch die Spielräume für Umverteilung gesunken sind. Letzteres erklärt, warum in sozial benachteiligten Gruppen Parteien Resonanz finden, die eine Schließung nationaler Grenzen und eine Renationalisierung von Macht fordern, während Vertreter von Wirtschaftsverbänden, aber auch von Umweltverbänden für Internationalisierung eintreten. Diese politische Restrukturierung scheint, wie Hanspeter Kriesi und Edgar Grande argumentierten, eine neue territoriale Konfliktlinie ${ }^{45} \mathrm{zu}$ erzeugen, die sich in regionalistischen Parteien und Bewegungen äußert. Diese Parteien entstanden im linken wie im rechten Spektrum, sie artikulieren Interessen an einer Umverteilung von Macht zwischen Ebenen. Tatsächlich beobachten wir aber eher eine Umkehrung von Verhältnissen in einer seit langem durch internationale Verflechtung geprägten politischen Strukturierung. Während im frühen 20. Jahrhundert sich die Arbeiterparteien international orientierten, verteidigen Sozialdemokraten heute nationale Sozialsysteme, wohingegen Verfechter neoliberaler Politik oder umweltpolitischer Regulierung auf transnationale Marktschaffung wie lokal differenzierte Lösungen setzen. 
Restrukturierungen der politischen Organisation von Gesellschaften lassen sich also kaum mit der Unterscheidung von Verstaatlichung oder Vergesellschaftung bzw. Privatisierung beschreiben. Sie fördern primär eine Ausdifferenzierung von Staatsfunktionen zwischen verschiedenen Ebenen, auf denen Staat und Private in vielen Aufgabenfeldern kooperieren. Dabei variieren die Formen der Kooperation. In der Tendenz werden Auseinandersetzungen um Sicherheit und um die Regulierung von Märkten zunehmend internationalisiert, Konflikte um soziale Sicherung und Umverteilung dagegen nach wie vor auf der Ebene des Nationalstaates ausgetragen, wenn sie nicht sogar regionalisiert bzw. kommunalisiert werden. Wenn sich in jüngster Zeit die Tendenz einer Kompetenzverlagerung auf private Leistungsanbieter abgeschwächt hat, darf dies nicht als Rückkehr des Staates interpretiert werden. Vielmehr zeigt sich darin nur eine neue Variante der Funktionsverschiebung, die sich auf der lokalen Ebene in anderer Weise vollzieht als auf nationaler oder internationaler Ebene. Kommunen scheinen inzwischen wieder ihr Engagement in öffentlichen Unternehmen entdeckt zu haben, Staaten setzen dagegen nach wie vor auf die Regulierung privater Leistungsanbieter und beschränken sich auf Gewährleistungsverantwortung; die EU begrenzt mit ihrer Politik der Liberalisierung im europäischen Binnenmarkt nach wie vor staatliche Interventionen. Ähnliche Folgen haben Restrukturierungen in der Sozialpolitik. Entgegen allen Unkenrufen kam es nicht zu einem Niedergang des Wohlfahrtsstaates. Damit sollen weder Einschnitte in Sozialleistungen, die der Konsolidierung der Finanzierungsbasis dienen, noch die Zunahme sozialer Ungleichheit, die primär durch ökonomische Entwicklungen verursacht sind, bestritten werden. Hinsichtlich der institutionellen Struktur des Wohlfahrtsstaates, auf die es hier ankommt, beobachten wir Differenzierungsprozesse mit der Folge, dass staatliche Leistungssysteme und private Versicherungen sich ergänzen (zum Beispiel private Zusatzrenten, Kinderbetreuung in Unternehmen). Indem sie arbeitsteilig Leistungen erbringen, werden staatliche und nichtstaatliche Organisationen auch in diesem Funktionsbereich zunehmend organisatorisch verflochten, weil Komplementarität zur Koordinierung von Angeboten und Standards zwingt. Die EU, die von vielen für einen Abbau sozialer Leistungen verantwortlich gemacht wird, hat ihre Regulierungsfunktion auf dieses Feld ausgedehnt und inzwischen Funktionen der Standardisierung und Kontrolle sozialer Leistungsstandards übernommen. ${ }^{46}$ Redistributive Sozialpolitik bleibt jedoch Aufgabe des Staates.

Wenn meine Beobachtungen richtig sind, wandelt sich der Staat durch eine Restrukturierung seiner äußeren Grenzen, also seiner »äußeren Form «. Dadurch entsteht weder ein Weltstaat, noch zerfallen etablierte moderne Staaten, vielmehr durchdringen sich Staaten sowie transnationale und subnationale Ebenen gegenseitig und sind mit privaten oder gesellschaftlichen Organisationen zunehmend verflochten. Äußere Grenzen von Gebieten, Nationen und Funktionsbereichen lösen sich nicht auf, weil sie in der Institutionenordnung des Staates verankert sind, aber sie werden durch gesellschaftliche Interaktionen konterkariert. Faktisch entstehen neue Grenzen, die die bestehenden Grenzen überlagern, teilweise innerhalb und

46 Ferrera 2005.

Leviathan, 40. Jg., 2/2012 
teilweise jenseits des Staates. ${ }^{47}$ Entgrenzung und Grenzziehung führen zu Differenzierungsprozessen des Politischen. Diese Prozesse, so vermute ich, betreffen die äußere Form des Staates ungleichgewichtig; sie tangieren die relativ starren Territorialstrukturen stärker als die Staatsbürgernation, diese aber wiederum stärker als die relativ flexibel definierbaren Staatsfunktionen. Die dadurch entstehende politische Ordnung, deren Konturen vor allem in Europa deutlich sichtbar sind, kann man als »verflochtenen, multinationalen Mehrebenenstaat» bezeichnen.

\section{Institutionelle Dynamik und Veränderungen der inneren Form des Staates}

Der Dynamik von Grenzüberschreitungen oder -verschiebungen und den Verflechtungen zwischen Staaten, zwischen Ebenen, auf denen Staatsgewalt ausgeübt wird, zwischen Nationen sowie zwischen dem Staat und nichtstaatlichen Organisationen steht eine relativ hohe Persistenz der internen Institutionen gegenüber. Das gilt jedenfalls, wenn man den »modernen "Staat in der sogenannten OECD-Welt betrachtet. Deswegen sollte man die Untersuchung des Wandels aber nicht auf die äußere Form des Staates beschränken, sondern die Anpassungen und durch Beharrungskräfte verursachten Spannungen in der inneren Form berücksichtigen.

Trotz aller Programme zur »Modernisierung des Staates « und trotz aller Bemühungen um institutionelle Reformen entwickeln sich Institutionen inkrementell und pfadabhängig. Das liegt an den Kausalmechanismen, die ihren Wandel bestimmen. Anders als gesellschaftliche Mechanismen, die die äußere Form des Staates tangieren, erfordern Veränderungen der inneren Form in der Regel explizit koordiniertes Handeln der betroffenen Akteure. Einseitige Handlungen können hier zwar Anpassungsreaktionen in den betroffenen Bereichen auslösen, institutionelle Reformen müssen hingegen mit allen Beteiligten ausgehandelt und dann durch Mehrheitsentscheidungen beschlossen werden. Zudem müssen reformierte Institutionen Anerkennung finden, das heißt kollektiven Leitideen entsprechen, mit denen sie legitimiert werden.

In Verhandlungen über eine Neuverteilung von Macht sind die Verteidiger des Status quo oft im Vorteil, weil Akteure, die Veränderungen erreichen wollen, sich gegenseitig blockieren, weil sie nicht dieselben Leitideen verfolgen oder weil sie die Notwendigkeit der Legitimation durch Leitideen nicht erkennen. Dies hat zur Folge, dass Leitideen, die in Reformdiskursen für Legitimation sorgen sollen, sich in Verfahren der Neuverteilung von Macht schwer durchsetzen lassen. Es bleiben daher Spannungen zwischen Ideen und Institutionen bestehen oder werden gar verschärft. In anderen Fällen können institutionelle Veränderungen aus kollektiven Praktiken hervorgehen, weil Akteure dazu tendieren, die Machtverteilung in bestehenden Institutionen durch strategisches Handeln zu ihren Gunsten zu verschieben. ${ }^{48}$ Die daraus resultierenden inkrementellen Entwicklungen wiederum können mit etablierten Leitideen in Widerspruch stehen. ${ }^{49}$ Man kann deshalb nicht erwarten, dass sich

47 Mau et al. 2008.

48 Mahoney, Thelen 2010.

49 Hesse, Benz 1990; Liebermann 2002. 
der Staat in allen seinen Dimensionen gleichmäßig und konsistent wandelt. Genauso wie die gesellschaftstheoretische Erklärung verweist also eine institutionentheoretische Erklärung auf Ungleichzeitigkeiten und Widersprüche.

Betrachten wir Veränderungen der internen Strukturen des Staates, dann stellen wir geradezu gegenläufige Entwicklungen fest: Verfassungsreformen und Verlagerungen von Kompetenzen fördern Europäisierung und Regionalisierung von Staatstätigkeit, bleiben aber hinter den Forderungen oder Leitideen teilweise weit zurück. Demokratische Institutionen des Nationalstaates werden ergänzt durch transnationale Institutionen der repräsentativen Demokratie, etwa das Europaparlament oder parlamentarische Versammlungen der NATO oder des Europarats, und lokale bzw. regionale Verfahren der partizipativen Demokratie. Letztere setzen voraus, dass auf diesen Ebenen autonome Entscheidungen getroffen werden können, was aber angesichts der vertikalen und horizontalen Politikverflechtung immer weniger zutrifft. Dagegen sind Exekutiven, also Regierungen und Bürokratien, zunehmend international verflochten, vor allem in ihrer Kooperation mit der EU-Kommission, aber auch in anderen internationalen Organisationen und internationalen Regimes. Dadurch nimmt ihre Selbstständigkeit gegenüber den sie kontrollierenden Organen zu, ohne dass diese Autonomie in Verfassungen legitimiert wäre. Zwar variieren diese Entwicklungen zwischen einzelnen Staaten erheblich, entsprechende Tendenzen sind aber generell zu beobachten.

(1) Die Verfassungsentwicklung der letzten Jahrzehnte betraf verschiedene Bereiche, angefangen von Grundrechten über Verfahren in demokratischen Institutionen bis zu Kompetenzordnung und Finanzverfassung im Verhältnis zwischen zentralen, regionalen und lokalen Untergliederungen des Staates. Es ist schwierig, in den diversen Änderungen ein bestimmtes Muster zu erkennen. Es fällt aber auf, dass Verfassungspolitik vorrangig auf die Veränderungen der territorialen Strukturen und die Ausdifferenzierung von Nationalitäten reagiert, die wir als Konsequenz gesellschaftlicher Entwicklungen beobachten. In vielen Staaten sind Kompetenzverlagerungen zwischen Ebenen festzustellen. In Europa resultieren diese aus der Integration von Staaten zu einer ökonomischen und politischen Union, ein Prozess, der in anderen Weltregionen erst begonnen hat. Innerhalb von Staaten, so haben empirische Untersuchungen festgestellt, gibt es Machtverschiebungen auf die regionale Ebene, die föderale und unitarische Staatsorganisationen gleichermaßen betreffen. ${ }^{50}$ Teilweise sind diese Verschiebungen funktionalistisch zu erklären, kommen also zustande, weil Regierungen oder Verwaltungen ihre Kompetenz zusammenlegen, um grenzüberschreitende Probleme zu bewältigen; teilweise ergeben sie sich aus »mikropolitischen « Strategien von Regierungen, die Kompetenzspielräume extensiv interpretieren und nutzen. Stabiler institutioneller Wandel erfordert jedoch eine Revision von Verfassungsnormen in besonderen Verfahren, die Verfassungen gegen einfache Änderungen schützen. Da in verfassungspolitischen Reformvorhaben in der Regel Akteure, deren Machtposition tangiert ist, entweder an der Verhandlung über Entscheidungsvorschläge beteiligt sind oder an der Ratifikation mitwirken und dann oft über Vetomacht verfügen, geraten Staaten in eine Situation, die mit der

50 Marks et al. 2008.

Leviathan, 40. Jg., 2/2012 
»Politikverflechtungsfalle « 51 vergleichbar ist: ${ }^{52}$ Weitreichende Reformen scheitern leicht, selbst wenn bestehende Strukturen effektives oder demokratisches Regieren behindern und selbst wenn sie durch kollektive Leitideen gestützt würden. Inkrementelle Verfassungsänderungen sind daher die Regel. ${ }^{53}$

Trotz dieser generellen Hemmnisse bei Verfassungsreformen lassen sich Veränderungstendenzen beobachten. Dabei sind zwischen dem Prozess der Internationalisierung bzw. der europäischen Integration und Prozessen der Regionalisierung bemerkenswerte Differenzen festzustellen, die die Dynamiken, die Legitimität und die Strukturbildung betreffen.

- Jenseits der Nationalstaaten wird die institutionelle Entwicklung in erster Linie durch sektorale Interessen vorangetrieben oder sie folgt einer funktionalistischen Logik in einzelnen Politikbereichen. Das gilt für die Europäische Union ebenso wie für andere Institutionen. Dagegen zielen Reformen der territorialen Verfassung von Nationalstaaten auf eine Neuverteilung von Macht zwischen Gebietskörperschaften. Sie rufen daher erhebliche Konflikte hervor, die oft den Charakter von "Nullsummenspielen " annehmen, bei denen die einen immer nur auf Kosten anderer gewinnen. Umfassende Reformen scheitern in der Regel oder enden mit nur begrenzten Veränderungen. Letztere werden durch die Dynamik der Internationalisierung und europäischen Integration gefördert, führen aber aufgrund von Machtkonflikten oft zu inkonsistenten Ergebnissen.

- Zwischenstaatliche Zusammenarbeit wie auch die Integration der europäischen Staaten zu einer "supranationalen « Union entwickeln sich bisher in einer Abfolge von Verträgen. Diese werden zwar durch nationale Parlamente ratifiziert, doch ohne dass sie in öffentlichen Diskursen legitimiert würden. Vielmehr resultieren sie aus der interstaatlichen Kooperation der Exekutiven. Die europäische Integration als demokratisch abgestimmtes Verfassungsprojekt scheiterte bekanntlich, doch der angestrebte Wandel wurde dadurch nicht blockiert, sondern durch intergouvernementale Verhandlungen bzw. transnationale Rechtsdiskurse und Rechtsprechung vorangebracht. Reformen, die auf eine Dezentralisierung der Staatsorganisation zielen, werden in der Regel als große Projekte angekündigt, die je nachdem, wie viele Konflikte sie nach sich ziehen, und je nach den gewählten Verfahrensweisen zu mehr oder weniger begrenzten Änderungen führen. ${ }^{54}$ Gemessen an den großen Zielen oder Ideen werden sie dann oft als Misserfolg bewertet. Die Verfassungsentwicklung verläuft auf dieser Ebene eher diskontinuierlich und in Widersprüchen zwischen Leitideen und faktisch durchsetzbaren Reformen.

- Die Verlagerung von staatlichen Kompetenzen auf supranationale Institutionen verstärkt die Verflechtung zwischen Ebenen, weil Staaten ihre Macht nur unter der Bedingung abgeben, dass sie Entscheidungen in den betreffenden Politikfel-

51 Scharpf 1985.

52 Benz 2011.

53 Vgl. Elkins et al. 2009.

54 Benz, Knüpling 2012. 
dern weiterhin beeinflussen können. Reformen der Binnenorganisation von Staaten zielen dagegen häufig darauf ab, die Autonomie der Regionen zu erweitern (etwa Verfassungsreformen in Belgien, Italien, Spanien oder im Vereinigten Königreich) und Politik zwischen den Ebenen zu entflechten (etwa Föderalismusreformen in Deutschland und in der Schweiz). ${ }^{55}$ Dies gilt vor allem in Fällen, in denen regionalistische Bewegungen eine Reform der territorialen Staatsorganisation vorantreiben. Auch insofern geraten institutionelle Veränderungen in einen Widerspruch zu bestimmten Leitideen. Die europäische Entwicklung wird mit Begriffen wie Verfassungssynthese ${ }^{56}$ und Verfassungsverbund ${ }^{57}$ beschrieben, die auf ein hohes Maß an Verflechtung der Ordnungen verweisen, Verfassungsreformen in Nationalstaaten zielen dagegen häufig auf eine erweiterte Autonomie regionaler Politik gegenüber dem Zentralstaat. Beide Prozesse sind schwerlich kompatibel.

Generell zeichnen sich die Staatsreformen der letzten Jahrzehnte also dadurch aus, dass sie gleichzeitig mehr Autonomie für die dezentralen Einheiten und mehr Koordination auf der supranationalen Ebene anstreben. Diese Ziele widersprechen sich, weil Dezentralisierung externe Effekte verursacht und daher mehr intergouvernementale Koordination erfordert und weil zunehmende Politikverflechtung zwischen Staaten und supranationalen Institutionen die regionale Autonomie einschränkt. Verfassungen, die eigentlich die institutionelle Ordnung von Staatlichkeit stabilisieren sollen, können diese Widersprüche nur in einer dauernden Evolution auflösen. Staaten gewinnen dadurch an Anpassungsfähigkeit, laufen aber Gefahr, dass Machtverteilungen instabil werden und nicht mehr legitimiert werden können. Verfassungen »verflüssigen « sich in Prozessen, die mehr durch Verfassungsgerichte und Exekutiven als durch demokratische Verfahren gesteuert werden.

(2) Genauso wie die Verfassung wandeln sich auch demokratische Institutionen vor allem in Anpassungsprozessen, die durch Machtverschiebungen in den Strukturen der Gewaltenteilung ausgelöst werden, weniger hingegen infolge von Reformen. Die Demokratie, wie sie im modernen Staat verwirklicht ist, bleibt im Kern an ein Territorium und einen Nationalstaat gebunden. Sie verändert sich durch Anlagerung von sektorspezifischen Formen partizipativer Verfahren, die auch in grenzüberschreitenden Kontexten Entscheidungen legitimieren können. In vielen Staaten stärkt die Mehrebenenverflechtung die Exekutive und fördert in parlamentarischen Regierungssystemen Tendenzen einer »Präsidentialisierung «. ${ }^{58}$ Innerhalb der EU wehren sich nationale Parlamente gegen diese Entwicklung, die hier durch die Einrichtung des Europäischen Parlaments nicht korrigiert werden konnte. Angesichts der Tatsache, dass Loyalitäten von Staatsbürgern zunehmend auch regionale oder kulturelle Diversität ausdrücken, ist das Subsidiaritätsprinzip zur Leitidee einer demokratischen Mehrebenenordnung geworden. Die damit verbundene Vorstellung,

55 Benz, Knüpling 2012.

56 Fossum, Menéndez 2011, S. 45-76.

57 Pernice 1999.

58 Poguntke, Webb 2005.

Leviathan, 40. Jg., 2/2012 
kollektives Handelns in kleineren Gemeinschaften würde die Partizipation der Bürger fördern, lässt sich aber angesichts der komplexen Aufgaben moderner Staaten kaum verwirklichen. Tatsächlich beobachten wir in Regionen und Kommunen eher die elitären Strukturen einer Verhandlungsdemokratie. Multinationale Staaten müssen zudem Minderheiten gegen die Mehrheitsherrschaft schützen, was ebenfalls konkordanzdemokratische Verfahren fördert. Die für die EU intensiv diskutierte Spannung zwischen Effektivität und demokratischer Legitimität des Regierens (bzw. zwischen »input- und output-Legitimität ${ }^{59}$ ) zeigt sich daher auch innerhalb von Staaten.

Allerdings läuft die Entwicklung in modernen Demokratien nicht auf eine lineare Machtverschiebung zugunsten der Exekutive hinaus, so wenig wie sie in eine Herrschaft von Partei- und Verbandseliten mündet. Etablierte demokratische Institutionen unterstützen vielmehr auch Gegenmächte gegen Eliten, wodurch die Zahl der effektiven Vetospieler steigt. Mechanismen wie Wahlen, öffentliche Diskurse oder der Parteienwettbewerb verlieren dadurch an Bedeutung gegenüber der Konfliktregelung in Verhandlungen oder der Dynamik von Macht und Gegenmacht. Aus normativer Sicht mag man die damit verbundenen Legitimitätsdefizite beklagen. Tatsache ist aber, dass die neuen Vetopunkte nur im institutionellen Kontext der auf Freiheit und Gleichheit der Bürger beruhenden repräsentativen Demokratie entstehen konnten, die nun in den fragmentierten gesellschaftlichen und politischen Strukturen des Mehrebenenstaates funktionieren muss. In der Form, die man als »komplexe Demokratie « bezeichnen kann, beruhen Schaffung und Begrenzung von Macht zwar nach wie vor auf der Institutionalisierung von Herrschaft durch Kompetenzzuweisungen an Ämter, sie ergeben sich aber auch aus ergänzenden Interaktionen zwischen korporativen Akteuren, zwischen solchen, die politische Ämter im Staat innehaben, und solchen, die private Interessen oder Interessen anderer Staaten vertreten. In diesem Kontext basiert Machtschaffung auf ausgehandelter Kooperation, Machtbegrenzung auf kompetitiven Beziehungen.

(3) Exekutiven und Bürokratien haben sich in den letzten Jahrzehnten erheblich verändert, wenn auch nicht in dem Maße oder so, wie es Protagonisten des New Public Management erhofft hatten. Die Welle von Verwaltungsreformen traf in den professionsorientierten Verwaltungen des angelsächsischen Raums eher auf Resonanz als in den organisationsorientierten Bürokratien Kontinentaleuropas. ${ }^{60}$ Sie sollten zunächst dazu dienen, Behörden durch Spezialisierung und Wettbewerb »effizienter « zu machen, später bemühte man sich um erweiterte Bürgerbeteiligung und verbesserte Koordination. Generell wurden Verwaltungsstrukturen aber an die Anforderungen einer wachsenden Verflechtung mit privaten und internationalen Organisationen angepasst. Indikatoren dafür sind neue unabhängige Agenturen (agencies, Regulierungsbehörden) auf nationaler oder europäischer Ebene, die spezielle Regulierungsaufgaben gegenüber öffentlichen und privaten Leistungsanbietern erfüllen, sektorale Netzwerke oder Kooperationsbeziehungen zwischen Behörden und Privaten vor allem im Bereich der technischen Infrastruktur sowie regionale und

59 Scharpf 1999.

60 Pollitt, Bouckaert 2004. 
lokale Netzwerke, welche die Wettbewerbsfähigkeit von Städten und Regionen verbessern sollen. Im Vergleich zu klassischen Bürokratien mögen solche Verwaltungen zwar effektiver sein, aber sie sind schwerer durch demokratische Organe zu kontrollieren, obwohl sie mehr als die traditionellen Vollzugsverwaltungen auch politische Entscheidungen treffen. Politisierung und Verselbstständigung der Verwaltung gehen also Hand in Hand.

Zusammengefasst kann man den Wandel der inneren Form des modernen Staates wie folgt charakterisieren: Während gesellschaftliche Mechanismen (grenzüberschreitende Mobilität, Loyalitätsbeziehungen, Widerspruch gegen politische Herrschaft - exit/entry, loyalty, voice) eine starke Veränderungsdynamik erzeugen und sich wechselseitig zu verstärken scheinen, treffen Veränderungsmechanismen im Innern oft auf Beharrungskräfte. Diese sind kollektivem Handeln in Institutionen immanent, aber mehr oder weniger stark ausgeprägt. Dementsprechend führt auch der Wandel in den Binnenstrukturen des Staates zu Inkonsistenzen zwischen den einzelnen institutionellen Dimensionen. Verfassungsreformen und Verfassungsentwicklungen förderten - oft entgegen der ihnen zugrunde liegenden Leitideen - eine vertikale Ausdifferenzierung der Staatsorganisation und bewirkten zugleich mehr Interdependenzen zwischen zentral und dezentral erfüllten Staatsaufgaben. Demokratie bleibt nach wie vor an ein Gebiet gebunden und somit innerhalb von Ebenen organisiert. Bemühungen von Parlamenten, ihre Regierungen auch in der Mehrebenenpolitik zu kontrollieren, oder Ansätze einer interparlamentarischen Verflechtung und transnationaler Vereinigungen von Parteien und Interessengruppen, die in der EU und in einzelnen Föderalstaaten zu beobachten sind, ändern daran nichts Grundsätzliches. Reformen von Bürokratien der letzten Jahrzehnte sollten der Effizienzsteigerung dienen, führten aber zu sektoraler Differenzierung, erweiterten die Autonomie von Verwaltungen und förderten teilweise vertikale Verwaltungsverflechtungen - um den Preis, dass die politische Kontrolle der Bürokratie durch demokratische Institutionen schwieriger wurde. Der Wandel der inneren Form des Staates verläuft also nicht in allen Dimensionen der institutionellen Ordnung gleichzeitig, in gleichem Maße oder in konsistenter Weise.

\section{Schluss}

Der moderne Staat wandelt sich, aber das war schon immer so. Dass er als Herrschaftsform kontinuierlich existiert, erklärt sich aus seiner Wandlungsfähigkeit. Schon Edmund Burke erkannte: Ein Staat ohne die Mittel für gewisse Veränderungen ist ohne Mittel für seine Bewahrung. ${ }^{61}$ Insofern kann man mit Fug und Recht bezweifeln, ob wir in einer Zeit der »Transformation « des Staates leben. Anders als es mit diesem Begriff suggeriert wird, verläuft der Wandel kontinuierlich, erfasst aber einzelne Dimensionen der staatlichen Institutionenordnung nicht gleichzeitig und jedenfalls nicht umfassend. So wenig, wie der Staat sich im Niedergang befun-

61 "A state without the means of some change is without the means of its conservation «; Burke 1993 [1790], S. 21.

Leviathan, 40. Jg., 2/2012 
den hat, so wenig beobachten wir nun seinen Wiederaufstieg. Machtverschiebungen sind, wie Uwe Schimank in einer differenzierungstheoretischen Argumentation begründet hat, ${ }^{62}$ in die widersprüchliche institutionelle Ordnung des modernen Staates eingeschrieben. Sie spiegeln das Spannungsverhältnis zwischen Machtschaffung und Machtbegrenzung wider.

Wenn man mit abstrakten Begriffen operiert, neigt man leicht zu Überzeichnungen einer Diskrepanz zwischen Begriff und Realität. Aus diesem Grund ist die Staatswissenschaft nicht völlig frei von den Einflüssen ideologischer Debatten in der Politik. Die Wirklichkeit ist aber komplizierter, und man darf mit dem Staatsbegriff die Komplexität nicht so stark reduzieren, dass man die Realität nicht mehr angemessen erfasst. Konzepte, die den Staat mit Souveränität oder Gewaltmonopol verbinden, die ihn als Apparat, Agentur oder korporativen Akteur betrachten, die ihn als Rechtsordnung oder als System verstehen wollen, haben immer seine Macht, seine Handlungsfähigkeit und seine Einheit entweder übertrieben oder unterschätzt. In jedem Fall sind sie nicht geeignet, den Wandel des Staates zu erfassen. Den Staat als institutionelle Ordnung zu begreifen, wie es hier vorgeschlagen wurde, lässt sich zum einen aus einer entwicklungsgeschichtlichen Analyse gut begründen, bietet zum anderen eine angemessene Grundlage dafür, den Wandel zu verstehen und zu erklären. Institutionen sind keine statischen Gebilde, sondern verändern sich ständig, sei es als Folge emergenter Effekte kollektiven Handelns oder als intendiertes oder unintendiertes Ergebnis von politisch beschlossenen Reformen. Institutionen sind ferner intern differenziert, und entsprechend verläuft der Wandel in verschiedenen Prozessen, die jeweils von Bereichen der Institution ausgehen und in anderen dann zeitlich versetzte, komplementäre oder gegenläufige Veränderungen auslösen können. Institutionen sind schließlich offen für Einflüsse von außen, die durch Interaktionen zwischen Akteuren über ihre Grenzen zustande kommen. Diese Eigenschaften des institutionalistischen Staatsverständnisses ermöglichen nicht nur, die Komplexität des Wandels zu beachten, sondern auch, sie zu erklären.

Den Staat als Institution zu bezeichnen bedeutet noch nicht, sich einer institutionentheoretischen Erklärung anzuschließen. Neoinstitutionalisten wie Vertreter des historischen Institutionalismus tendieren dazu, zu erklären, warum Wandel verhindert wird und warum Institutionen eine hohe Persistenz aufweisen. ${ }^{63}$ Veränderungen müssen gegen diese Beharrungskräfte durchgesetzt werden, und dies erreichen Akteure, die über die Macht verfügen, Regeln der institutionellen Ordnung zu umgehen oder zu verändern. Macht, Veränderungen zu induzieren, haben vor allem jene Akteure, die Grenzen überschreiten, die eine Institution nach außen abgrenzen, sowie Akteure, die innerhalb von Institutionen Widersprüche zwischen Regeln oder zwischen Regeln und Leistungserwartungen ausnutzen können. Institutionalistische Ansätze müssen also um Theorien kollektiven Handelns, um eine akteurszentrierte Perspektive erweitert werden.

Ein akteurszentrierter Institutionalismus macht auf zwei Dynamiken aufmerksam, die den Wandel des Staates maßgeblich prägen. Zum einen leben wir in einer

62 Schimank 2009.

63 Mahoney, Thelen 2010, S. 6. 
Gesellschaft, deren Strukturen zunehmend verflüssigt werden und dadurch Akteuren mehr Optionen bieten, bisherige Bindungen durch eine feste Struktur zu überschreiten. Dies betrifft auch den Staat. Seine Gebietsgrenzen, die Definition und Integration seiner Bürgerschaft sowie die funktionale Begrenzung seiner Macht werden durch mobile Akteure und durch Transaktionen, durch Differenzierung von Loyalitäten und durch veränderte Organisation kollektiver Interessen in Frage gestellt und verlieren im Verhältnis zu neuen Grenzziehungen an Gewicht. Begriffe wie Entgrenzung, Denationalisierung oder Souveränitätsverlust beschreiben diese Entwicklungen allerdings unzureichend, vielmehr ist von einer zunehmenden Interdependenz zwischen Staaten, einer Pluralisierung von Nationen und einer funktionalen Arbeitsteilung und Machtverflechtung zwischen staatlichen und gesellschaftlichen Akteuren auszugehen. All dies stellt den Staat vor Herausforderungen, aber schwächt ihn nicht unbedingt im Vergleich zu früheren Zeiten. Die zweite Dynamik zeigt sich in den internen Strukturen, die durch Machtverschiebungen und ausgehandelte Reformen verändert werden. Sie wird durch Beharrungskräfte von Institutionen gebremst, in einzelnen Bereichen mehr als in anderen.

In diesen Prozessen wandelt sich der Staat und entwickelt sich zu einem verflochtenen, multinationalen Mehrebenensystem. Der Kern der Problematik liegt dabei weniger in einer sinkenden Macht von Regierungen, autonom über kollektive Angelegenheiten der Staatsbürger entscheiden zu können, und er liegt auch nicht primär in einer Aushöhlung des Sozialstaates. Vielmehr liegt er in der generellen Tatsache, dass die Anpassungsfähigkeit des Staates überfordert werden kann, weil einerseits Leitideen institutioneller Reformen von Verfassungen, Demokratie und Bürokratie nicht oder nur partiell geeignet sind, die internen Strukturen an externe Herausforderungen anzupassen, und weil andererseits kollektive Prozesse bei einer zunehmenden Zahl von Vetospielern immer mit einer erheblichen Diskrepanz zwischen Zielen und erreichten Veränderungen enden müssen.

Es soll hier nicht behauptet werden, dass diese knappe Skizze den Wandel des Staates angemessen beschreibt. Eines kann aber festgehalten werden: Die theoretischen Überlegungen sowie die Hinweise auf reale Veränderungen, die die Theorie illustrieren sollten, zeigen eher eine Kontinuität des Wandels als Prozesse der Transformation, des Zerfall oder der Revitalisierung des Staates. Die wirkliche Entwicklung ist weniger dramatisch, als es publikumswirksame Beiträge gerne darstellen. Aber dies sollte uns nicht dazu veranlassen, den Wandel des Staates einfach hinzunehmen oder als normal zu erklären. Nüchterne sozialwissenschaftliche Analysen können auf widersprüchliche Entwicklungen aufmerksam machen, welche die Stabilität und Leistungsfähigkeit des Staates gefährden können. Sie können aber auch den souveränen, autonomen, unitarischen Nationalstaat sowie den Markt schaffenden, dezentralisierten Minimalstaat als Staatsideologien zurückweisen, die in den vergangenen drei Jahrzehnten die Staatspolitik zu problematischen Reformen verleitet haben. 


\section{Literatur}

Bartolini, Stefano 2005. Restructuring Europe. Centre formation, system building and political structuring between the nation state and the European Union. Oxford: Oxford University Press.

Benz, Arthur 2008. Der moderne Staat. Grundlagen der politologischen Analyse. 2., überarbeitete Aufl. München: Oldenbourg.

Benz, Arthur 2011: "Escaping joint-decision traps: national and supranational experiences compared ", in The EU's decision traps: comparing policies, hrsg. v. Falkner, Gerda, S. 199-216. Oxford: Oxford University Press.

Benz, Arthur; Knüpling, Felix. Hrsg. 2012: Changing federal constitutions. Lessons from international comparison. Opladen, Berlin, Toronto: Barbara Budrich Publishers.

Brenner, Neil 2004. New state spaces: urban governance and the rescaling of statehood. Oxford: Oxford University Press.

Bull, Hedley 1977. The anarchical society: a study of order in world politics. London: Macmillan.

Burke, Edmund 1993 [1790]. Reflections on the revolution in France. Oxford: Oxford University Press.

de Jouvenel, Bertrand 1989. On power. The national history of its growth. Indianapolis: Liberty Fund.

Dyson, Kenneth 1980. The state tradition in Western Europe. Oxford: Marin Robertson.

Elias, Norbert 1976. Über den Prozeß der Zivilisation. Soziogenetische und psychogenetische Untersuchungen. Bd. 2: Wandlungen der Gesellschaft. Entwurf zu einer Theorie der Zivilisation. Frankfurt a. M.: Suhrkamp.

Elkins, Zachary; Ginsburg, Tom; Melton, James 2009. The endurance of constitutions. Cambridge: Cambridge University Press

Evans, Peter B.; Rueschemeyer, Dietrich; Skocpol, Theda. Hrsg. 1985. Bringing the state back in . Cambridge: Cambridge University Press.

Falletti, Tulia; Lynch, Julia 2009. »Context and causal mechanisms in political analysis ", in Comparative Political Studies 42, S. 1143-1166.

Ferrera, Maurizio 2005. The boundaries of welfare. European integration and the new spatial politics of social protection. Oxford: Oxford University Press.

Fossum, John-Erik; Menéndez, Augustino José 2011. The constitutional gift. A constitutional theory for a democratic European Union. Lanham u.a.: Rowman \& Littlefield.

Grimm, Dieter 2009. Souveränität. Herkunft und Zukunft eines Schlüsselbegriffs. Berlin: Berlin University Press.

Habermas, Jürgen 2006. Die postnationale Konstellation: Politische Essays. 5. Aufl. Frankfurt a. M.: Suhrkamp.

Heinze, Rolf G. 2009. Rückkehr des Staates? Politische Handlungsmöglichkeiten in unsicheren Zeiten. Wiesbaden: VS Verlag für Sozialwissenschaften.

Hesse, Joachim Jens; Benz, Arthur 1990. Die Modernisierung der Staatsorganisation. BadenBaden: Nomos.

Hurrelmann, Achim et al. 2008. "Die Zerfaserung des Nationalstaates: Ein analytischer Rahmen ", in Zerfasert der Nationalstaat? Die Internationalisierung politischer Verantwortung, hrsg. v. Hurrelmann, Achim et al., S. 21-52. Frankfurt a. M., New York: Campus.

Immergut, Ellen 1998. »The theoretical core of the new institutionalism «, in Politics and Society 26, S. 5-34.

Isensee, Joseph 1995. »Staat «, in Staatslexikon, Bd. 5, hrsg. v. der Görres-Gesellschaft, S. 34-157. Freiburg, Basel, Wien: Herder.

James, Harold 2009. Die Krise der Finanzmärkte und die Rückkehr des Staates. TransState Working Papers 85. Bremen: Sfb 597 »Staatlichkeit im Wandel«.

Jellinek, Georg 1905. Allgemeine Staatslehre. 2. Aufl. Berlin: Verlag von O. Häring.

Keohane, Robert O. 2000. "Sovereignty in international society ", in The global transformation reader. An introduction to the globalization debate, hrsg. v. Held, David; McGrew, Anthony, S. 109-123. Cambridge: Blackwell.

Kriele, Martin 2003. Einführung in die Staatslehre. 6. Aufl. Stuttgart: Kohlhammer. 
Kriesi, Hanspeter et al. 2006. »Globalization and the transformation of the national political space: six European countries compared «, in European Journal of Political Research 45, S. 921-956.

Leibfried, Stephan; Zürn, Michael 2006 a. »Von der nationalen zur post-nationalen Konstellation ", in Transformation des Staates?, hrsg. von Leibfried, Stephan; Zürn, Michael, S. 19-65. Frankfurt a. M.: Suhrkamp.

Leibfried, Stephan; Zürn, Michael. Hrsg. 2006 b. Transformation des Staates? Frankfurt a. M.: Suhrkamp.

Liebermann, Robert 2002. »Ideas, institutions, and political order: explaining political change «, in American Political Science Review 96, S. 697-712.

Luhmann, Niklas 1981. Politische Theorie im Wohlfahrtsstaat. München: Olzog.

Mahoney, James 2000. "Path dependence in historical sociology ", in Theory and Society 29, S. 507-548.

Mahoney, James; Thelen, Kathleen 2010. "A Theory of Gradual Institutional Change «, in Explaining institutional change. Ambiguity, agency, and power, hrsg. v. Mahoney, James; Thelen, Kathleen, S. 1-37. Cambridge: Cambridge University Press.

Maíz, Ramón; Requejo, Ferran. Hrsg. 2005. Democracy, nationalism and multiculturalism. London, New York: Frank Cass.

Mann, Michael 1993. The sources of power. Vol. II: The rise of classes and nation-states, 1760-1914. Cambridge: Cambridge University Press.

Marks, Gary; Hooghe, Liesbet; Schakel, Arjan H. 2008. »Patterns of Regional Authority «, in Regional and Federal Studies 18, S. 167-181.

Mau, Steffen et al. 2008. »Grenzen in der globalisierten Welt. Selektivität, Internationalisierung, Exterritorialisierung ", in Leviathan 36, 1, S. 123-148.

Mayntz, Renate 2010. "Die Handlungsfähigkeit des Nationalstaats bei der Regulierung der Finanzmärkte", in Leviathan 38, S. 175-187.

Miller, David 1995. On nationality. Oxford: Clarendon Press.

Möllers, Christoph 2008. Der vermisste Leviathan. Staatstheorie der Bundesrepublik. Frankfurt a. M.: Suhrkamp.

Offe, Claus 1972. Strukturprobleme des kapitalistischen Staates. Frankfurt a. M., New York: Campus.

Offe, Claus 1987. »Die Staatstheorie auf der Suche nach ihrem Gegenstand. Beobachtungen zur aktuellen Diskussion ", in Jabrbuch zur Staats- und Verwaltungswissenschaft, hrsg. v. Ellwein, Thomas et al., S. 309-320. Baden-Baden: Nomos.

Offe, Claus 1996. Modernity and the state: east, west. Cambridge: MIT Press.

Orren, Karen; Skowronek, Stephen 2004. The search for American political development. Cambridge: Cambridge University Press.

Pernice, Ingolf 1999. »Der Europäische Verfassungsverbund auf dem Wege der Konsolidierung «, in Jahrbuch des öffentlichen Rechts der Gegenwart 48, S. 206-231.

Pierson, Paul 2004. Politics in time. History, institutions and social analysis. Princeton: Princeton University Press.

Poguntke, Thomas; Webb, Paul 2005. The presidentialization of politics: a comparative study of modern democracies. Oxford: Oxford University Press.

Pollitt, Christopher; Bouckaert, Geert 2004. Public management reform: a comparative analysis. 2. Aufl. Oxford: Oxford University Press.

Reinhard, Wolfgang 1999. Geschichte der Staatsgewalt. Eine vergleichende Verfassungsgeschichte Europas von den Anfängen bis zur Gegenwart. München: C. H. Beck.

Rokkan, Stein 1999. State formation, nation-building, and mass politics in Europe. The theory of Stein Rokkan. Based on his collected works. Oxford: Oxford University Press.

Roth, Klaus 2003. Genealogie des Staates. Prämissen des neuzeitlichen Politikdenkens. Berlin: Duncker \& Humblot.

Scharpf, Fritz W. 1985. »Die Politikverflechtungsfalle: Europäische Integration und deutscher Föderalismus im Vergleich «, in Politische Vierteliahresschrift 26, S. 323-356.

Scharpf, Fritz W. 1999. Governing in Europe: effective and democratic? Oxford: Oxford University Press.

Schimank, Uwe 2005. »Weltgesellschaft und Nationalgesellschaften: Funktionen von Staatsgrenzen ", in Weltgesellschaft - Theoretische Zugänge und empirische Problemlagen. Son-

Leviathan, 40. Jg., 2/2012 
derheft der Zeitschrift für Soziologie, hrsg. v. Heintz, Bettina; Münch, Richard; Hartmann, Tyrell, S. 394-414. Stuttgart: Lucius \& Lucius.

Schimank, Uwe 2009. " Vater Staat‘: ein vorhersehbares Comeback. Staatsverständnis und Staatstätigkeit in der Moderne", in Der moderne Staat 2, S. 249-269.

Schuppert, Gunnar Folke 2009. Staat als Prozess. Eine staatstheoretische Skizze in sieben Aufzügen. Frankfurt a. M., New York: Campus.

Spruyt, Hendrik 1994. The sovereign state and its competitors. Princeton: Princeton University Press.

Tilly, Charles 1990. Coercion, capital, and European states, AD 900-1990. Oxford: Blackwell. Wagner, Peter 1996. "Der Soziologe als Übersetzer «, in DIE ZEIT, Nr. 15, 5. April 1996, S. 34. Weber, Max 1976. Wirtschaft und Gesellschaft. Tübingen: Mohr.

Wiener, Antje; Diez, Thomas 2009. European integration theory. 2. Aufl. Oxford: Oxford University Press.

Willke, Helmut 1997. Die Supervision des Staates. Frankfurt a. M.: Suhrkamp.

Winkler, Heinrich August 2009. Geschichte des Westens: Von den Anfängen in der Antike bis zum 20. Jahrhundert. München: C. H. Beck.

Zürn, Michael 1998. Regieren jenseits des Nationalstaates. Frankfurt a. M.: Suhrkamp. 
Zusammenfassung: Die Staatsdiskussion ist von Thesen über dessen Aufstieg oder Niedergang geprägt. Differenzierte Aussagen über den Wandel des Staates finden sich dabei selten. Das liegt oft bereits an einem Staatsbegriff, der für die Erklärung des Wandels nicht geeignet ist. Gesellschaftszentrierte wie institutionalistische Theorien liefern bislang wenig überzeugende Grundlagen, die behauptete Transformation des Staates angemessen zu erklären. Ausgehend von dieser Kritik wird in diesem Beitrag ein analytischer Rahmen skizziert, der zum besseren Verständnis des Wandels des modernen Staates beitragen soll. Er lenkt den Blick auf ungleichzeitige Entwicklungen und dadurch verursachte strukturelle Inkonsistenzen. Während die Staaten sich in verflochtene multinationale Mehrebenensysteme wandeln, zeichnen sich ihre Institutionen der Verfassung, Demokratie und Bürokratie durch ein beträchtliches Beharrungsvermögen aus.

Stichworte: Staat, Verfassung, Demokratie, Bürokratie, Staatstheorie, Wandel des Staates

\section{Decline or Revival? Reflections on the evolution of the state}

Summary: In academic discussions about the state, scholars often emphasize its rise or decline. Rarely do we find differentiated hypotheses on the evolution of the state. Theoretical concepts are rarely appropriate to studies of how and why states change. Society-centred and institutionalist approaches cannot convincingly explain the supposed transformation of the state. Based on this critique, the article outlines an analytical framework that seeks to contribute to a better understanding of the evolution of the modern state. It draws attention to uncoordinated developments and, accordingly, structural inconsistencies. While states have turned into interlinked, multinational, multilevel systems, their internal institutions (constitution, democracy and bureaucracy) reveal a significant inertia.

Keywords: state, constitution, democracy, bureaucracy, state theory, transformation of the state

Autor

Prof. Dr. Arthur Benz

Technische Universität Darmstadt

FB 2, Institut für Politikwissenschaft

Residenzschloss

D-64283 Darmstadt

Tel. 06151/16-2842

Email: abenz@pg.tu-darmstadt.de

Leviathan, 40. Jg., 2/2012 